\title{
Thermodynamic Properties of Liquids Including Solutions. XV. Intramolecular Contacts and Their Effects on Excess Enthalpies of Solutions of Chain Molecules, Especially Benzene Solutions of Normal Alkanes and Polymethylenes
}

\author{
Maurice L. Huggins* and John W. KenNedY** \\ *135 Northridge Lane, Woodside, California 96062, U.S.A. \\ **Institute of Polymer Science, University of Essex, Colchester C04 3SQ, England.
}

(Received August 7, 1978)

\begin{abstract}
The theory of excess enthalpy $\left(H^{\mathrm{E}}\right)$ and enthalpic component of the interaction parameter $\left(\chi_{\mathrm{h}}\right)$, dealt with in previous papers of this series, is extended to allow for the effects of intramolecular contacts in chain molecule solutes. The number of these contacts, as a function of the number of mers in the chain molecules and their concentration in the solution, is estimated from enumerations over a class of self-avoiding walks (paths) in a lattice-graph. Applied to solutions of polymethylenes in benzene, for which the equilibrium constant parameter $(K)$ has been previously estimated to be unity, we find intramolecular contacts to have little effect on the excess enthalpy at $25^{\circ} \mathrm{C}$ even for long chains. The enthalpic part of the interaction function is predicted to depend strongly on the extent of intramolecular contacting. Extensions of the theory to solutions for which $K \neq 1$ are briefly considered.

KEY WORDS Thermodynamics of Solutions / Enthalpy / Interaction Function / Benzene / Alkanes / Polymethylene / Oligomer / Polymer Solutions / Intramolecular Contacts / Self-Avoiding Walks / Lattice Graphs / Graph Theory /
\end{abstract}

The statistical mechanical theory of the thermodynamic properties of liquids, which is the subject of this series of papers, ${ }^{1-4}$ was recently applied $^{5}$ to the published excess enthalpy data for solutions of normal alkanes in benzene at $25^{\circ} \mathrm{C}$. This theory leads to an expression for the excess enthalpy of mixing $\left(H^{E}\right)$ in terms of the mole fractions $x_{1}$ (benzene) and $x_{2}$ (alkane) of the two components

$$
\begin{gathered}
H^{\mathrm{E}}=\varepsilon_{\Delta} x_{1} z_{2} g_{\mathrm{K}} \\
z_{2}=1-z_{1}=\frac{r_{\sigma} x_{2}}{x_{1}+r_{o} x_{2}} \\
g_{\mathrm{K}}=2\left[\left(1+K^{\prime} z_{1} z_{2}\right)^{1 / 2}-1\right] / K^{\prime} z_{1} z_{2} \\
K^{\prime}=4\left(K^{-1}-1\right)
\end{gathered}
$$

Here, $\varepsilon_{\Delta}$ is an "exchange energy" for replacing contacts $(1-1,2-2)$ between like molecules by contacts (1-2) between unlike molecules. The parameter $r_{\sigma}$ is the ratio of the numbers of contact sites per molecule in each of the two types

$$
r_{o}=\sigma_{2}{ }^{0} / \sigma_{1}{ }^{0}
$$

$K$ is an equilibrium constant, measuring the relative probabilities of contacts of the three types. $K=1$ signifies that the contacts are random in the sense that their relative numbers depend only on the relative numbers of contact sites of the two kinds (hence, on the concentration). If $K=1$, then $g_{\mathrm{K}}=1$ and eq 1 to 4 simplify to

$$
H^{\mathrm{E}}=\frac{\varepsilon_{\Delta} r_{\sigma} x_{1} x_{2}}{x_{1}+r_{\sigma} x_{2}}
$$

By fitting three points on each of the empirical smoothing curves reported ${ }^{6-10}$ for excess enthalpies of mixing benzene with various $n$-alkanes, Huggins ${ }^{5}$ obtained values for the three statistical mechanical parameters $\left(K, \varepsilon_{\Delta}, r_{\sigma}\right)$.

Comparison of the calculated parameters for the ten systems showed that the set

$$
K=1 ; \varepsilon_{\Delta}=3068 \mathrm{~J} \mathrm{~mol}^{-1} ; r_{\sigma}=0.2(n+1)
$$

represents the data within the rather large experi- 
mental uncertainty, (as judged by the standard deviations of data points from their smoothing curves and the differences between data reported for the same system by different investigators).

In this way, the 30 to 40 empirical parameters required by the experimenters' smoothing curves are replaced by just three theoretically meaningful global parameters that can also be used to predict excess enthalpies for benzene solutions of $n$-alkanes for which measurements are not yet available.

The excess enthalpy is defined as the excess of the enthalpy of one mole (Avogadro's number of molecules) of the mixture over what it would be if each molecule contributed the same amount of enthalpy as in its pure liquid. In a pure $n$-alkane liquid, with $n$ greater than 5 , there must be some intramolecular contacts. In a solution for which the parameter $K$ equals 1 , there should be the same average number of such contacts per alkane molecule as in the pure alkane liquid. The excess enthalpy therefore measures only the intermolecular contacts. The actual values of $\sigma_{\text {al sane }}^{0}$ and $r_{\sigma}$ derived from excess enthalpy measurements, should not show a rectilinear dependence on the number of carbon atoms in the molecule, as implied by eq 7 . Since the number of intramolecular contacts is likely to be relatively small for the alkanes for which $H^{E}$ data have been published, the deviation from rectilinearity for these solutions would be expected to be very small. The published data are not accurate enough to enable the extent of these deviations to be determined.

In this paper we employ enumerations over a class of walks in a lattice-graph, together with the excess enthalpy data just discussed, to estimate the chain length and concentration dependence of the intra- and intermolecular contacts, and also the excess enthalpy and the enthalpic contribution to the interaction parameter of benzene solutions of polymethylene oligomers (normal alkanes) and higher polymers.

\section{STATISTICAL ANALYSIS OF THE EXCESS ENTHAPY DATA}

As a first step, we have re-estimated the three parameters $\left(K, \varepsilon_{\Delta}\right.$, and $\left.r_{\sigma}\right)$ for the benzene solutions of $n$-alkanes with $n$ equal to 5 through 17 , for which excess enthalpy data have been published.
Direct-global least-squares fits to eq 1, with all data points equally weighted (regardless of differences in reliability at different concentrations and by different experimenters using different apparatus), suggest that the parameters of eq 7 be refined to the optimal set

$$
K=1 ; \varepsilon_{\Delta}=3170 \mathrm{~J} \mathrm{~mol}^{-1} ; r=0.192(n+1)
$$

The standard deviation, based on the (computed experimental) residuals of $H^{\mathrm{E}}$, is $27 \mathrm{~J} \mathrm{~mol}^{-1}$, for 263 data points whose mean is $885 \mathrm{~J} \mathrm{~mol}^{-1}$.

Future experiments of greater accuracy will doubtless lead to further refinements of the parameters. (For other comparable systems, $H^{\mathrm{E}}$ data of considerably greater accuracy have been published; see for example Huggins ${ }^{2}$ ). Extension of the range of $n$-alkanes studied to larger $n$ values will also be useful in estimating departures from the rectilinear dependence of $r_{\sigma}$ on $n$.

\section{ESTIMATION OF INTRAMOLECULAR CONTACTS BY LATTICE WALK ENUMERATIONS}

In previous papers of this series dealing with polymer solutions, ${ }^{3,4}$ the distinction between intra- and intermolecular contacts has been neglected. This neglect, which affects the concentration and chain-length dependence of the thermodynamic properties of polymer solutions, we now explore.

Experimental data appropriate for the calculation of the numbers of intramolecular and intermolecular contacts in a solution as functions of concentration and the number $(n)$ of mers in the polymer, are not available. However, combinatorial problems relating to chain configurations can be usefully approximated by appropriate enumerative results defined on walks in a tetrahedral (diamond) lattice-graph. ${ }^{11}$ For the case at hand, we expect the mean number of intramolecular contacts to be proportional to the mean number (per walk) of first-neighbour approaches $^{12}\langle\mathrm{~J}\rangle$, taken over all $(n-1)$-step selfavoiding walks (called "paths" in graph theory ${ }^{13}$ ) in the diamond lattice-graph.

Such walks have been categorised in great detail $^{14}$ for $n=2$ to 20 , using a fast exact enumeration program developed from the "list method" of Martin ${ }^{15}$ for the Essex University Computer 
Thermodynamic Properties of Liquids. XV.

System. Each walk is categorised as having $n$ points, $i$ self-intersections, $j$ first-neighbour approaches, and $t$ trans steps. For each category the number of walks, $W(n, i, j, t)$ and some statistical measures, such as the mean square end-toend distance, are tabulated.

For paths (self-avoiding walks), $i=0$. Disregrading the possibility of trans/gauche weighting, we write

$$
W(n, j) \equiv \sum_{t} W(n, 0, j, t)
$$

and then

$$
\langle\mathrm{J}\rangle_{n}=\frac{\sum_{j} j W(n, j) \mathrm{e}^{-\alpha(j)}}{\sum_{j} W(n, j) \mathrm{e}^{-\alpha(j)}}
$$

where $\alpha(j)$ is a weighting function for $j$ firstneighbour approaches. If $K=1$, it is appropriate to set $\alpha(j)=0$. This gives us the unibiassed mean for $n$-point paths, $\langle\mathrm{J}\rangle_{n}{ }^{0}$. The number of contact sites per $n$-alkane chain used in intramolecular contacts is then proportional to $2\langle\mathrm{~J}\rangle_{n}{ }^{0}$. The total number of contact sites per $n$-alkane chain is proportional $^{5}$ to $2(n+1)$. Hence, the fraction of chain contact sites used in intramolecular contacts is

$$
f_{n}=\langle\mathrm{J}\rangle_{n}{ }^{0} /(n+1)
$$

The mean numbers $\langle\mathbf{J}\rangle_{n}{ }^{0}$ derived from our lattice walks tables and the fractions $f_{n}$ are listed in Table I.

Jain and Pruitt, ${ }^{16}$ working from Theorem 1 of Dvoretzky and Erdös, ${ }^{17}$ showed that the number of self-intersections made by an (unbiassed) walk of $N$ steps in a (diamond) lattice-graph is an asymptotically normally distributed variable with mean $\langle\mathrm{I}\rangle_{N}{ }^{0}$ and variance $\operatorname{Var}\left(\mathrm{I}_{N}{ }^{0}\right)$ given by

$$
\begin{aligned}
\langle\mathrm{I}\rangle_{N}{ }^{0} & =c_{1} N+c_{2} N^{1 / 2} \\
\operatorname{Var}\left(\mathrm{I}_{N}{ }^{0}\right) & =c_{3} N \ln N
\end{aligned}
$$

where $c_{1}, c_{2}, c_{3}$ are constants related to the asymptotic return probability. Exploiting the apparent parallelism ${ }^{18}$ between walks of $N$ steps and paths of $N-1$ steps, we conjecture a similar asymptotic behaviour for the mean number $\langle\mathrm{J}\rangle_{n}{ }^{0}$. Consequently,

$$
\langle\mathrm{J}\rangle_{n}{ }^{0}=k_{1} n+k_{2} n^{1 / 2}
$$

It is stressed by Fisher and Sykes ${ }^{19}$ that convergence to asymptotic behaviour is rapid (especially for paths), so that data from exact enumeration on relatively short walks furnish highly reliable
Table I. Mean numbers $\langle\mathbf{J}\rangle_{n}{ }^{0}$ of intramolecular contacts per molecule and fractions $\left(f_{n}\right)$ of sites making intramolecular contacts

\begin{tabular}{cccc}
\hline \multirow{2}{*}{$n$} & \multicolumn{2}{c}{$\begin{array}{c}\text { Exact values from } \\
\text { tabulated walks }\end{array}$} & $\begin{array}{c}\text { Calculated from } \\
\text { eq 15 and 16 }\end{array}$ \\
\cline { 2 - 4 } & $\langle\mathrm{J}\rangle_{n}{ }^{0}$ & $f_{n}$ & $f_{n}$ \\
\hline 6 & 0.07407 & 0.01058 & 0.00892 \\
7 & 0.10127 & 0.01266 & 0.01355 \\
8 & 0.15451 & 0.01717 & 0.01741 \\
9 & 0.19912 & 0.01991 & 0.02068 \\
10 & 0.26135 & 0.02376 & 0.02350 \\
11 & 0.30638 & 0.02553 & 0.02596 \\
12 & 0.36769 & 0.02828 & 0.02813 \\
13 & 0.41828 & 0.02988 & 0.03006 \\
14 & 0.47952 & 0.03197 & 0.03179 \\
15 & 0.53245 & 0.03328 & 0.03336 \\
16 & 0.59397 & 0.03494 & 0.03479 \\
17 & 0.64855 & 0.03603 & 0.03609 \\
18 & 0.71020 & 0.03738 & 0.03729 \\
19 & 0.76615 & 0.03831 & 0.03839 \\
20 & 0.82781 & 0.03942 & 0.03942 \\
30 & & & 0.04654 \\
50 & & & 0.05410 \\
100 & & & 0.06152 \\
500 & & & 0.07126 \\
$10^{3}$ & & & 0.07354 \\
$10^{6}$ & & & 0.07875 \\
$\infty$ & & & 0.07892 \\
\hline
\end{tabular}

estimates of the parameters in asymptotic expressions of this sort. Accordingly, we use the results for $n=10$ through 20 from Table I to determine the least-squares optimum values.

$$
k_{1}=0.07891 ; \quad k_{2}=-0.1678
$$

with a correlation coefficient of 0.998 . From eq 11 and 14 it follows that for longer chains

$$
f_{n}=\frac{k_{1} n+k_{2} n^{1 / 2}}{n+1}
$$

and approaches 0.07891 as $n$ tends to infinity. Values of $f_{n}$ calculated from this equation are given in Table I for comparison with the exact values for the shorter paths.

It is worth emphasising that the foregoing analysis is not altered in essential details by varying the choice of lattice-graph in which selfavoiding walks are constructed. Our results are not at all dependent on any geometric features that may be associated with an embedding of the lattice-graph in space. ${ }^{20}$ 
The analysis can clearly be extended to cover cases with $K$ not equal to unity by employing an appropriate weighting function $\alpha(j)$ in eq 10, thereby obtaining a suitably biassed mean $\langle\mathbf{J}\rangle_{n}$. Equations 12 to 14 apply to the unbiassed distribution of walks (for which $\alpha(j)=0$ ). From the parallelism mentioned above, the unbiassed distribution is expected to be asymptotically normal with mean $\langle\mathbf{J}\rangle_{n}{ }^{0}$ and variance $\operatorname{Var}\left(J_{n}{ }^{0}\right)$. If the set of paths is now biased by a weighting factor $\beta$ per first-neighbour approach, i.e.,

$$
\alpha(j)=\beta^{j}
$$

then the new (biassed) asymptotic mean is given approximately (see Gordon, et al. ${ }^{18}$ ) by

$$
\langle\mathbf{J}\rangle_{n}=\langle\mathbf{J}\rangle_{n}{ }^{0}-\operatorname{Var}\left(J_{n}{ }^{0}\right)
$$

Hence, when $K \neq 1$

$$
\langle\mathbf{J}\rangle_{n}=k_{1} n+k_{2} n^{1 / 2}-k_{3} n \ln n
$$

We hope to return to this relation in future papers. For the remainder of this paper we restrict ourselves to the case $K=1$.

\section{CORRELATION OF LATTICE WALK RESULTS WITH EXCESS ENTHALPY DATA}

According to the theory being applied the parameter $r_{\sigma}$ determines the skewness of the excess enthalpy $v s$. concentration curve. ${ }^{2,4,5}$ Assuming the number of contact sites per mer to be independent of the chain length and in the absence of intramolecular polymer-polymer contacts, $r_{\sigma}$ is a rectilinear function of $n$ as assumed in eq 7 and 8 . Since, as has been explained, the excess enthalpy is, by definition, a function of the ratio $\left(r_{\sigma}\right)$ of the number of polymer sites making intermolecular contacts to the number of solvent sites making (intermolecular) contacts, the observed $r_{\sigma}$ values are expected to depart from a rectilinear relation as the chain length increases.

Assuming that the fraction $\left(f_{n}\right)$ of polymer contact sites making intramolecular contacts is given by eq 15 and 16 ,

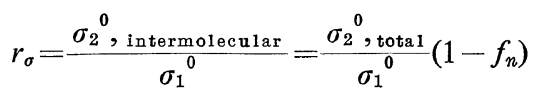

a fit of the published $H^{\mathrm{E}}$ data for benzene solutions of the lower $n$-alkanes furnishes the globally optimal parameter values ( $c f$. eq 8$)$.

$$
K=1 ; \varepsilon_{\Delta}=3175 \mathrm{~J} \mathrm{~mol}^{-1} ; r_{\sigma}=0.196(n+1)\left(1-f_{n}\right)
$$

Table II compares the values of $r_{\sigma}$ computed from eq 8 with those from eq 21. (For $n>6$, differences in $r_{\sigma}$ resulting from use of the asymptotic result for $f_{n}$ in place of the exact values given in Table $I$ are insignificant). Both sets agree, to within the probable limits of certainty, with values obtained by individually fitting each

\begin{tabular}{|c|c|c|c|}
\hline \multirow{2}{*}{$n$} & \multirow{2}{*}{$\begin{array}{c}r_{\sigma}=0.192 \\
(n+1) \\
(\text { eq 8) }\end{array}$} & \multicolumn{2}{|c|}{$r_{\sigma}=0.196(n+1)\left(1-f_{n}\right)$} \\
\hline & & $\begin{array}{l}\text { Using exact } \\
f_{n} \text { (Table I) }\end{array}$ & $\begin{array}{c}f_{n} \text { from } \\
\text { eq } 15 \text { and } 16\end{array}$ \\
\hline 5 & 1.152 & 1.152 & 1.172 \\
\hline 6 & 1.344 & 1.357 & 1.360 \\
\hline 7 & 1.536 & 1.548 & 1.546 \\
\hline 8 & 1.728 & 1.734 & 1.733 \\
\hline 9 & 1.920 & 1.921 & 1.919 \\
\hline 10 & 2.112 & 2.105 & 2.105 \\
\hline 11 & 2.304 & 2.292 & 2.291 \\
\hline 12 & 2.496 & 2.476 & 2.476 \\
\hline 13 & 2.688 & 2.662 & 2.661 \\
\hline 14 & 2.880 & 2.846 & 2.846 \\
\hline 15 & 3.072 & 3.032 & 3.031 \\
\hline 16 & 3.264 & 3.216 & 3.215 \\
\hline 17 & 3.456 & 3.401 & 3.400 \\
\hline 18 & 3.648 & 3.585 & 3.584 \\
\hline 19 & 3.840 & 3.770 & 3.769 \\
\hline 20 & 4.032 & 3.952 & 3.953 \\
\hline 30 & 5.952 & & 5.793 \\
\hline 50 & 9.792 & & 9.453 \\
\hline 100 & 19.39 & & 18.58 \\
\hline 500 & 96.20 & & 91.20 \\
\hline $10^{3}$ & 192.2 & & 181.8 \\
\hline $10^{8}$ & $1.92 \times 10^{5}$ & & $1.81 \times 10^{5}$ \\
\hline
\end{tabular}
$n$-alkane-benzene system over the range $(n=5$ through 17) for which experimental data are available.

Table II. Calculated contact ratios

Figure 1 displays representative plots of $H^{\mathrm{E}}$ as a function of $x_{2}$, calculated from eq 6 . The dashed lines were drawn for parameters from eq 8 (assuming a rectilinear dependence of $r_{\sigma}$ on $n$, with no intramolecular contacts). The full lines are for the parameters of eq 21. Clearly, the differences are negligible relative to the scatter of the experimental points (also shown). The differences between the two curves for $n=50$ and 
Thermodynamic Properties of Liquids. XV.

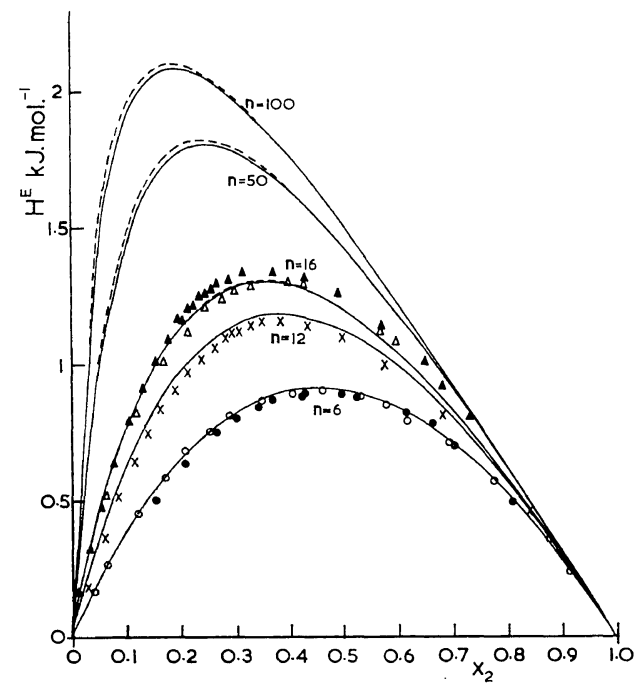

Figure 1. Representative excess enthalpy curves for normal alkanes in benzene at $25^{\circ} \mathrm{C}$, calculated from eq 6. The dashed lines are calculated using the parameters from eq 8 which were inferred on the assumption that all contacts are intermolecular. The full lines are calculated using the parameters of eq 21 obtained on the assumption that both intermolecular and intramolecular contacts participate, the extent of the latter having been estimated from enumerations over paths in a lattice-graph. Comparison with the data points:

for $n=6$, (O) Romani and Paz-Andrade ${ }^{7}$ and (O) Harris and Dunlop ${ }^{9}$

for $n=12$, ( $\times$ ) Diaz Pena and Menduina ${ }^{6}$

for $n=16$, (A) Diaz Pena and Menduina ${ }^{6}$

and $(\triangle)$ Lundberg and Grolier. ${ }^{10}$

suggests that even for large $n$ (polymethylene) the contribution from intramolecular contacts will be difficult to detect by directly analysing $H^{\mathrm{E}}$ data at fixed temperature.

between the two curves for $n=100$ suggest that, even for long alkanes, the effect of intramolecular contacting on $H^{\mathrm{E}}$ is not large. It seems unlikely, therefore, that measurements of the concentration dependence of $H^{\mathrm{E}}$ for $n$-alkanes in benzene (even if much more accurate than those that have been made) will be useful in estimating the extent of intramolecular contacting. We shall now discuss, however, a much more powerful way of studying this effect.

\section{THE ENTHALPY COMPONENT OF THE INTERACTION FUNCTION}

In treating the thermodynamic properties of solutions of linear polymers, ${ }^{3,4}$ it is usual to express concentrations as volume fractions $\left(\varphi_{1}\right.$, $\left.\varphi_{2}\right)$ rather than mole fractions $\left(x_{1}, x_{2}\right)$. It is also useful to examine the magnitude and concentration dependence of the interaction function $(\chi)$ and its enthalpic and entropic components $\left(\chi_{\mathrm{h}}, \chi_{\mathrm{s}}\right)$. The enthalpic component is closely related to the excess enthalpy. For mixtures with $K=1$ it is given by the relation ${ }^{3}$

$$
\chi_{\mathrm{h}}=\left(\frac{\varepsilon_{\Delta}}{R T}\right)\left[\frac{r_{\sigma / v}}{1+\left(r_{\sigma / v}-1\right) \varphi_{2}}\right]^{2}
$$

with

$$
r_{\sigma / v} \equiv\left(\frac{V_{1}}{V_{2}}\right) r_{\sigma}
$$

At $25^{\circ} \mathrm{C}$,

$$
\begin{gathered}
R T=8.3144 \times 298.15=2478.94 \mathrm{~J} \mathrm{~mol}^{-1} \\
V_{1}=89.406 \mathrm{~cm}^{3} \mathrm{~mol}^{-1}
\end{gathered}
$$

and

$$
V_{2}=\left(16.39 n+31.75-6 / n+90 / n^{2}\right) \mathrm{cm}^{3} \mathrm{~mol}^{-1}
$$

where eq 26 agrees with the molar volumes published by Rossini, et al., ${ }^{21}$ for $n=4$ through 8 , with an average deviation of less than 0.005 .

For the purposes of the ensuing discussion we shall use the values for $\varepsilon_{\Delta}$ and $r_{\sigma}$ as furnished by eq 8 when we neglect the effect of intramolecular contacts or eq 21 when we account for them. We are not here proposing to debate the question of whether or not chains exhibit intramolecular contacts, but rather to use the extreme case where there are no intramolecular contacts to assess the strength of the dependence of $\chi_{\mathrm{h}}$ on the extent of intramolecular contacting. Plots of $\chi_{\mathrm{h}}$ (Figures 2 and 3 ) for the two cases show surprisingly large differences, especially for small $\varphi_{2}$ and large $n$. Although the quantitative detail of these curves is something of an artifact of the parameter values (eq $8,21,26$ ), the marked qualitative differences between the two cases do allow contributions from the various parameters to be resolved so that the extent of intramolecular contacting can be meaningfully probed in some depth from experiments aimed at measuring $\chi_{\mathrm{h}}$ directly. For example, one can calculate $\chi_{\mathrm{h}}$ from the temperature coeffi- 


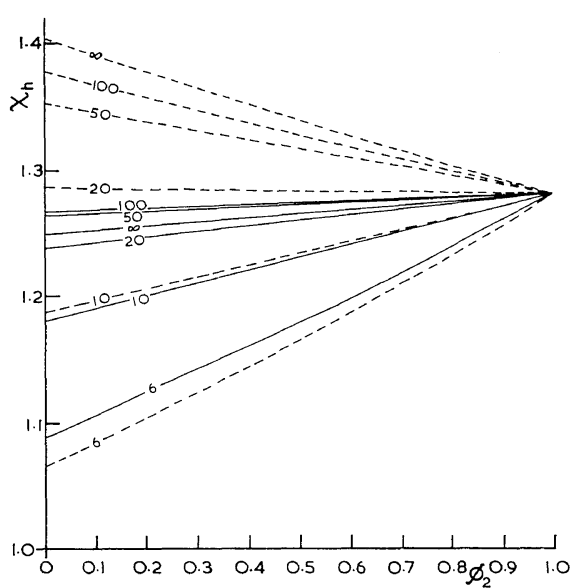

Figure 2. Enthalpic part of the interaction function, $\chi_{\mathrm{h}}$ vs. $\varphi_{2}$, calculated for representative $n$-alkanes in benzene show a marked dependence on the extent of intramolecular contacting. Dashed curves assume no intramolecular contacts, solid curves assume intramolecular contacts to an extent estimated from enumerating walks on lattice-graphs. Differences between the two sets of curves cannot be entirely attributed to uncertain parameter values obtained from fitting crude data for small $n$-alkanes, but represent significant dependence on the extent of intramolecular contacts. Compare also Figure 3.

cient of the interaction function $\chi$, using

$$
\chi_{\mathrm{h}}=-T^{2} \frac{\partial}{\partial T}(\chi / T)
$$

The parameter $\varepsilon_{\Delta}$ merely serves to ordinate-scale $\chi_{\mathrm{h}}$ (eq 22) and has the same effect whether intramolecular contacts are neglected or not. At $\varphi_{2}=1$ (see eq 22), $\chi_{\mathrm{h}}\left(=\varepsilon_{\Delta} / R T\right)$ is independent of $n$ and the influence of intramolecular contacts vanishes. Since for the two cases $\chi_{\mathrm{h}}=1.279$ and 1.281, respectively (when $\varphi_{2}=1$ ), the small difference in $\varepsilon_{\Delta}$ resulting from reoptimisation (compare eq 8 with eq 21) has an insignificant effect even in scaling $\chi_{\mathrm{h}}$.

For all $n$, the effect of intramolecular contacts on $\chi_{\mathrm{h}}$ increases with decreasing $\varphi_{2}$, attaining its maximum as $\varphi_{2} \rightarrow 0$, when

$$
\chi_{\mathrm{h}}=\left(\frac{\varepsilon_{\Delta}}{R T}\right) r_{\sigma / v}^{2}
$$

In the limit $n \rightarrow \infty$ we have, from eq 26, $V_{2} \sim$ $16.39 n$, while

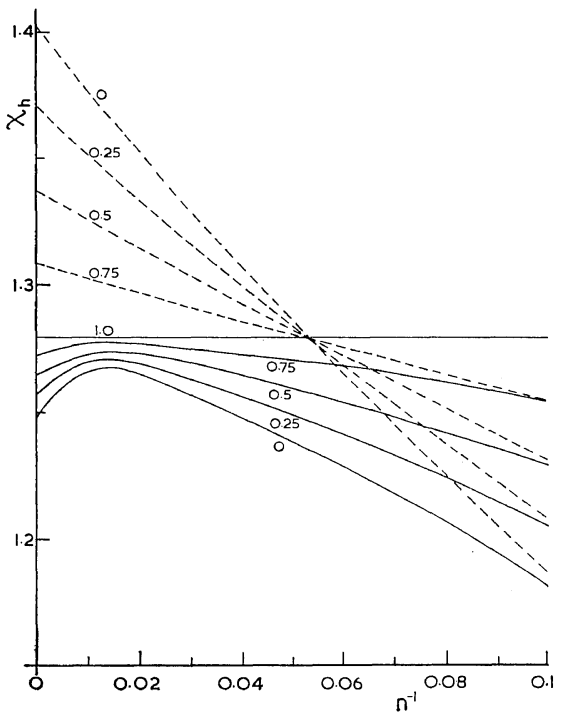

Figure 3. Enthalpic interaction function $\chi_{\mathrm{h}}$ vs. $n$ for various volume fractions $\varphi_{2}$ as shown on figure. Dashed and solid lines have the same significance as in Figures 1 and 2 . The marked differences between the two sets of curves make $\chi_{\mathrm{h}}$ a sensitive probe for the extent of intramolecular contacts (see text) since it separates the roles played by various parameters lifted from optimisation sets.

$$
\begin{aligned}
r_{\sigma} & =0.192(n+1) \sim 0.192 n \\
r_{\sigma / v} & \sim 1.04734 \\
\chi_{\mathrm{h}} & \sim \frac{1.40271}{\left(1+0.04734 \varphi_{2}\right)^{2}}
\end{aligned}
$$

when intramolecular contacts are absent; or

$$
\begin{aligned}
r_{\sigma} & =0.196(n+1)\left(1-f_{n}\right) \sim 0.196\left(1-k_{1}\right)_{n} \\
r_{\sigma / v} & \sim 0.98461 \\
\chi_{\mathrm{h}} & \sim \frac{1.24161}{\left(1-0.01539 \varphi_{2}\right)^{2}}
\end{aligned}
$$

when intramolecular contacts are counted. Comparison of eq 29 to 31 with eq 32 to 34 shows how the rather small effect of intramolecular interactions on $r_{\sigma}$ (or $r_{\sigma / v}$ ) is amplified in $\chi_{\mathrm{h}}$. Were this merely an amplification of small differences in parameters obtained by optimisation over scattered data (eq 8, 21, 26), it would have dubious value. However, $V_{2}$ plays an identical role in both cases and the major contribution stems from the better determined value of the parameter $k_{1}$ (see eq 15,16 ). 
The same point is even more forcefully demonstrated in relation to the existence of maxima displayed by $\chi_{\mathrm{h}}$ in Figure 3 . Using the case $\varphi_{2}=0$ as an example, it follows from eq 28 that $\partial \chi_{\mathrm{h}} / \partial n=0$ when

$$
V_{2} \frac{\partial r_{\sigma}}{\partial n}-r_{\sigma} \frac{\partial V_{2}}{\partial n}=0
$$

When intramolecular contacts are neglected let $r=p(n+1)$ for some parameter $p(=0.192$ in eq 8$)$. Equation 35 then requires that

$$
V_{2}-(n+1) \frac{\partial V_{2}}{\partial n}=0
$$

Substitution from eq 26 yields a cubic equation in $n$ which has no real positive solution. Thus the maximum in $\chi_{\mathrm{h}}$ is not an artifact of either the expression (eq 26) for $V_{2}$ or the parameter $p$ which does not even feature in eq 36 . When we allow for intramolecular contacts, let

$$
r=p(n+1)\left(1-f_{n}\right)=p\left\{1+\left(1-k_{1}\right) n-k_{2} n^{1 / 2}\right\}
$$

Then eq 35 requires that

$$
\begin{aligned}
& V_{2}\left\{1-k_{1}-\frac{1}{2} k_{2} n^{-1 / 2}\right\} \\
& -\left\{1+\left(1-k_{1}\right) n-k_{2} n^{1 / 2}\right\}\left(\frac{\partial V_{2}}{\partial n}\right)=0
\end{aligned}
$$

in which, again, the parameter $p$ does not appear, but which now suggests the existence of a maximum in $\chi_{\mathrm{h}}$ at $n \simeq 90$.

\section{DISCUSSION}

Quantitative models for the dependence of thermodynamic functions on concentration and molecular chain length are important for our understanding of the behaviour of polymer solutions. $^{22} \mathrm{~A}$ model which is already proven ${ }^{2}$ to describe the excess enthalpy of mixing $H^{\mathrm{E}}$ at fixed temperature of solutions of short chain molecules is not expected to apply to longer chain (polymer) molecules because of the occurrence of intramolecular contacts. The work reported here attempts to remedy this by estimating the extent of intramolecular contacting from enumerations over the class of self-avoiding walks in a tetrahedral lattice-graph.

When, as for solutions of $n$-alkanes in benzene, the contact distribution parameter $K$ is unity, we find that the extent of intramolecular contacting (as adduced from the unbiassed distribution of self-avoiding walks) has only a small effect on the enthalpy of mixing even for long chain molecules.

On the other hand, for the same systems, our analysis predicts a very sensitive dependence of the enthalpic component of the interaction function $\chi_{\mathrm{h}}$ on the extent of intramolecular contacting. Regrettably, direct measurements of this quantity for benzene- $n$-alkane (polymethylene) solutions are not yet available. Clearly such data would be of considerable theoretical importance in interpreting the extent of intramolecular contacts and the use of lattice-graph models for this phenomenon. Certainly this is an essential step toward better understanding the higher derivatives of the Gibbs free energy of mixing function to which so much of polymer technology is related. ${ }^{22}$

Acknowledgement. We thank Essex University for extensive use of its computer system. One of us (JWK) acknowledges support from the Science Research Council of the United Kingdom.

\section{REFERENCES}

1. M. L. Huggins, J. Phys. Chem., 74, 371 (1970); 75, 1255 (1971).

2. M. L. Huggins, J. Phys. Chem., 80, 1317, 2732 (1976); 81, 2136 (1977).

3. M. L. Huggins, Polymer, 12, 357 (1971).

4. M. L. Huggins, Polym. J., 4, 502 (1973).

5. M. L. Huggins, Br. Polym. J., 9, 189 (1977).

6. M. Diaz Pena and C. Menduina, J. Chem. Thermodyn., 6, 387 (1974).

7. L. Romani and M. I. Paz-Andrade, Int. DATA Ser., Selec. Data Mixtures, Ser. A, 100 (1973).

8. H. W. Schnaible, H. C. Van Ness, and J. M. Smith, AIChE J., 3, 147 (1957).

9. K. R. Harris and P. J. Dunlop, J. Chem. Thermodyn., 2, 805 (1970).

10. G. W. Lundberg, J. Chem. Eng. Data, 9, 193 (1964); J.-P. E. Grolier, Int. DATA Ser., Selec. Data Mixtures, Ser. A, 223 (1974).

11. C. Domb, Chap. 1 in "Phase Transitions and Critical Phenomena," Vol. 3, C. Domb and M. S. Green, Ed., Academic Press, New York N.Y., 1974.

12. M. E. Fisher and B. J. Hiley, Phys. Rev., 114, 45 (1959).

13. F. Harary, "Graph Theory", Addison-Wesley, Reading, Mass., 1969. 
14. J. W. Kennedy, unpubl. results; lists available on request.

15. J. L. Martin, Proc. Camb. Phil. Soc., 58, 92 (1962).

16. N. C. Jain and W. E. Pruitt, J. Analyse Math., 24, 369 (1970).

17. A. Dvoretzky and P. Erdös, "Proc. Second Berkeley Symposium on Mathematical Statistics and Probability," University of California Press, Berkeley, California, 1950, p 353.

18. M. Gordon, J. A. Torkington, and S. B. Ross-
Murphy, Macromolecules, 10, 1090 (1977).

19. M. E. Fisher and M. F. Sykes, Phys. Rev., 114, 45 (1959).

20. D. S. McKenzie, Phys. Rep., 27, 35 (1976).

21. F. D. Rossini, et al., "Selected Values of Properties of Hydrocarbons and Related Compounds," API Research Project 44, 1956, Table $20 \mathrm{~b}$.

22. M. Gordon, P. Irvine, and J. W. Kennedy, 5th Prague Discussion Conference (1976); Pure Appl. Chem., in press. 